\title{
Recurrence and Metastasis of Lung Cancer Demonstrate Decreased Diffusion on Diffusion-Weighted Magnetic Resonance Imaging
}

\author{
Katsuo Usuda ${ }^{1 *}$, Motoyasu Sagawa ${ }^{1}$, Nozomu Motomo ${ }^{1}$, Masakatsu Ueno ${ }^{1}$, \\ Makoto Tanaka ${ }^{1}$, Yuichiro Machida ${ }^{1}$, Sumiko Maeda ${ }^{1}$, Munetaka Matoba ${ }^{2}$, Hisao \\ Tonami $^{2}$, Yoshimichi Ueda ${ }^{3}$, Tsutomu Sakuma ${ }^{1}$
}

\begin{abstract}
Background: Diffusion-weighted magnetic resonance imaging (DWI) is reported to be useful for detecting malignant lesions. The purpose of this study is to clarify characteristics of imaging, detection rate and sensitivity of DWI for recurrence or metastasis of lung cancer. Methods: A total of 36 lung cancer patients with recurrence or metastasis were enrolled in this study. While 16 patients underwent magnetic resonance imaging (MRI), computed tomography (CT) and positron emission tomography-computed tomography (PET-CT), 17 underwent MRI and CT, and 3 underwent MRI and PET-CT. Results: Each recurrence or metastasis showed decreased diffusion, which was easily recognized in DWI. The detection rate for recurrence or metastasis was $100 \%(36 / 36)$ in DWI, 89\% (17/19) in PET-CT and 82\% (27/33) in CT. Detection rate of DWI was significantly higher than that of CT $(p=0.0244)$ but not significantly higher than that of PET-CT $(p=0.22)$. When the optimal cutoff value of the apparent diffusion coefficient value was set as $1.70 \times 10^{-3} \mathrm{~mm}^{2} / \mathrm{sec}$, the sensitivity of DWI for diagnosing recurrence or metastasis of lung cancer was $95.6 \%$. Conclusions: DWI is useful for detection of recurrence and metastasis of lung cancer.
\end{abstract}

Keywords: Lung cancer - recurrence - metastasis - diagnosis - magnetic resonance imaging - diffusion-weighted imaging

Asian Pac J Cancer Prev, 15 (16), 6843-6848

\section{Introduction}

Diffusion-weighted magnetic resonance imaging (DWI) exploits the random, translational motion, or so-called Brownian movement, of water molecules in biologic tissue (Le Bihan et al., 1988). Recently, DWI has been used to detect the restricted diffusion of water molecules in the body. The primary use of DWI has been in brain imaging, mainly for the evaluation of intracranial tumors, acute ischemic stroke and demyelinating diseases (Tien et al., 1994; Sorensen et al., 1996). Diffusion of water molecules in malignant tumors is generally restricted compared to that in normal tissue, resulting in a decreased apparent diffusion coefficient (ADC) value (Szafer et al., 1995; Takahara et al., 2004).

MRI examination carries no risk of exposure to radiation, and the examination can be done in every hospital whereas hospitals equipped with PET-CT are limited because of the potential risk in handling the radioisotope of ${ }^{18} \mathrm{~F}$-FDG. Furthermore, MRI examination is relatively more affordable compared to a PET-CT examination. In terms of patient well-being patients do not have to fast before the DWI examination, do not need exogenous contrast medium, and less time is required for the examination.

However, it is unclear whether DWI is useful for detection of recurrence or metastasis of lung cancer and what the characteristics of recurrence or metastasis of lung cancer are in DWI. The purpose of this study is to clarify the characteristics, detection rate and sensitivity of DWI of recurrence and metastasis of lung cancer.

\section{Materials and Methods}

\section{Eligibility}

The study protocol for examining DWI in patients with lung cancer was approved by the ethical committee of Kanazawa Medical University (the approval number: No.189). Informed consent to a general clinical practice for MRI was obtained from each patient after discussing the risks and benefits of the examinations. MRI, CT and/or PET-CT were performed within 2 weeks in each patient. 


\section{Patients}

The clinical prospective study started in May 2009. Patients who underwent surgery for lung cancer and whose later recurrence sites were diagnosed histopathologically through bronchoscopy, percutaneous biopsy, or sputum cytology, or were diagnosed radiographically were enrolled in this study. There were 36 lung cancer patients with recurrence or metastasis from December 2009 to November 2013. Concerning postoperative pathological staging at operation, there were 4 Stage IA, 11 Stage IB, 2 Stage IIA, 4 Stage IIB, 13 Stage IIIA and 2 Stage IIIB. For gender, 26 were male and 10 were female. There were 19 adenocarcinomas, 15 squamous cell carcinomas, 1 carcinoid and 1 carcinosarcoma.

\section{Magnetic resonance imaging (MRI)}

All MRIs were obtained with a $1.5 \mathrm{~T}$ superconducting magnetic scanner (Magnetom Avanto; Siemens, Erlangen, Germany) with two anterior six-channel body phasedarray coils and two posterior spinal clusters (six-channels each). The conventional MRIs consisted of a coronal T1weighted spin-echo sequence and coronal and axial T2weighted fast spin-echo sequences. Diffusion-weighted magnetic resonance imagings (DWIs) using a single-shot echo-planar technique were performed under SPAIR (spectral attenuated inversion recovery) with respiratory triggered scan with the following parameter: TR/TE/flip angle, 3000-4500/65/90; diffusion gradient encoding in three orthogonal directions; $b$ value $=0$ and $800 \mathrm{~s} / \mathrm{mm}^{2}$; field of view, $350 \mathrm{~mm}$; matrix size, $128 \times 128$. After image reconstruction, a 2-dimensional (2D) round or elliptical region of interest (ROI) was drawn on the lesion which was detected visually on the ADC map with reference to the T2-weghted image or CT by the radiologist (H.T.) with 39 years of MRI experience who was unaware of the patients' clinical data. Areas with necrosis were excluded from the ADC measurement. The procedure was repeated three times and the minimum ADC value was obtained. The radiologist (H.T.) and one pulmonologist (K.U.) with 30 years of experience evaluated the MRI data. A consensus was reached if there were any differences of opinion. The optimal cutoff value (OCV) for diagnosing malignancy in DWI was determined to be $1.70 \times 10^{-}$ ${ }^{3} \mathrm{~mm}^{2} / \mathrm{sec}$ using receiver operating characteristics curve as reported in the previous article (Usuda et al., 2013). Recurrence or metastasis from lung cancers with an ADC value of the same or less than the OCV was defined as positive. Recurrence or metastasis from lung cancers with an ADC value of more than the OCV or those that could not be detected on DWI was defined as negative.

\section{Positron emission tomography - computed tomography (PET-CT)}

PET-CT scanning was performed with a dedicated PET camera (SIEMENS Biograph Sensation 16, Erlangen Germany) before surgery. All patients fasted for 6 hours before scanning. The dose of 18-fluoro-2-deoxy-glucose $\left({ }^{18} \mathrm{~F}-\mathrm{FDG}\right)$ administered was $3.7 \mathrm{MBq} / \mathrm{Kg}$ of body weight. After a 60-min uptake period, an emission scan was acquired for 3 min per bed position and a whole-body scan was performed on each patient using several bed positions according to the height of each patient. After image reconstruction, a 2-dimensional (2D) round region of interest (ROI) was drawn on a slice after visual detection of the highest count on the fused CT image by the radiologist (M.T.) with 14 years of radioisotope scintigraphy and PET-CT experience who was unaware of the patients' clinical data. The maximum standardized uptake value (SUVmax) was calculated as the ${ }^{18} \mathrm{~F}-\mathrm{FDG}$ accumulation within lesions.

\section{Computed tomography (CT)}

CT examinations were performed by using a multidetector row CT scanner, SOMATOM Definition Flash (SIEMENS, Forchheim, Germany). The instrument parameters at multidetector CT: Lung $(120 \mathrm{KV}, 160 \mathrm{~mA}$, 16x0.75-mm collimation, and 5-mm section thickness reconstruction, window level of -500 Hounsfield unit (HU), window width of $1500 \mathrm{HU})$, mediastinum (160 $\mathrm{mA}$, window level of $30 \mathrm{HU}$, window width of $400 \mathrm{HU}$ ), brain $(300 \mathrm{~mA}$, window level of $37 \mathrm{HU}$, window width of $90 \mathrm{HU})$ and abdomen $(240 \mathrm{~mA}$, window level of $30 \mathrm{HU}$, window width of $280 \mathrm{HU})$.

\section{Detection rate of recurrence or metastasis of lung cancer by examination}

For detection rate of recurrence or metastasis by examination, it was determined whether examinations were helpful or unhelpful in each case through discussion among doctors.

\section{Statistical analysis}

Statistical analysis was performed using StatView for Windows (Version 5.0; SAS Institute Inc. Cary, NC, USA). The data is expressed as the mean \pm standard deviation. Differences of ratios between two groups were compared by using the $\chi 2$ test. A P value of $<0.05$ was considered statistically significant.

\section{Results}

Sixteen patients underwent MRI, CT and PET-CT, 17 underwent MRI and CT, and 3 underwent MRI and PETCT. In the 36 lung cancer patients, recurrent or metastatic lesions of 12 patients were confirmed with pathological diagnosis. In the 24 patients whose recurrent or metastatic lesions were diagnosed radiographically, 7 patients died of lung cancer and 17 were alive with recurrence or metastasis as of June 2014.

Of the 36 lung cancer patients with recurrence or metastasis, recurrent or metastatic sites were: lung in 21 , lymph nodes in 10 , pleural cavity in 4 , bone in 4 , liver in 4 , brain in 1 and spleen in 1 (Total number of evaluated sites: 45) (Table 1).

Pulmonary metastasis was detected in the right lung (Figure 1). It was recognized by CT, PET-CT and DWI. Pulmonary metastasis was presented as decreased diffusion in DWI. Usually in DWI, heart, blood vessels, bronchus and benign lesions would not show decreased diffusion, and would not likely be detected. Pulmonary metastasis was recognized by decreased diffusion in DWI (Figure 2). Local recurrence in a residual lung was 
Recurrence and Metastasis of Lung Cancers Demonstrate Decreased Diffusion on Diffusion-Weighted MRI

Table 1. ADC and Sensitivity by DWI

\begin{tabular}{llll}
\hline $\begin{array}{c}\text { Recurrent or } \\
\text { metastatic } \\
\text { sites }\end{array}$ & $\begin{array}{c}\text { Number } \\
\text { of lesions }\end{array}$ & ADC & $\begin{array}{c}\text { Sensitivity } \\
\text { by DWI }\end{array}$ \\
\hline Lung & 21 & $1.31 \pm 0.278$ & $90.4 \%(19 / 21)$ \\
Lymph node & 10 & $1.36 \pm 0.171$ & $100 \%(10 / 10)$ \\
Pleura & 4 & $1.10 \pm 0.149$ & $100 \%(4 / 4)$ \\
Bone & 4 & $1.06 \pm 0.267$ & $100 \%(4 / 4)$ \\
Liver & 4 & $1.11 \pm 0.238$ & $100 \%(4 / 4)$ \\
Brain & 1 & 0.989 & $100 \%(1 / 1)$ \\
Spleen & 1 & 1.55 & $100 \%(1 / 1)$ \\
Total & 45 & $1.31 \pm 0.243$ & $95.6 \%(43 / 45)$ \\
\hline
\end{tabular}

*ADC: apparent diffusion coefficient, DWI: diffusion-weighted imaging

a
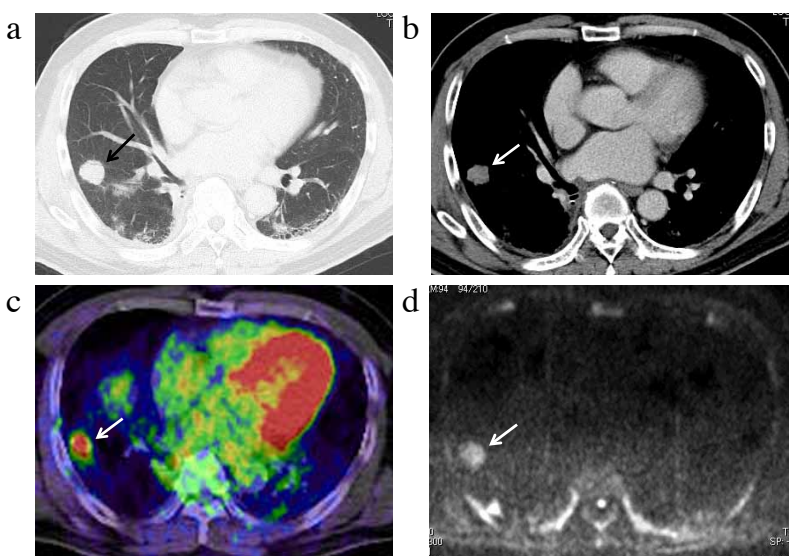

Figure 1. Case 1: A 65 Year-Old Male, Adenocarcinoma, Pulmonary Metastasis in Right Lower Lobe; a: CT (based on lung), b: CT (based on mediastinum), c: PETCT, SUVmax 8.34, d: DWI, ADC $1.265 \times 10^{-3} \mathrm{~mm}^{2} / \mathrm{sec}$
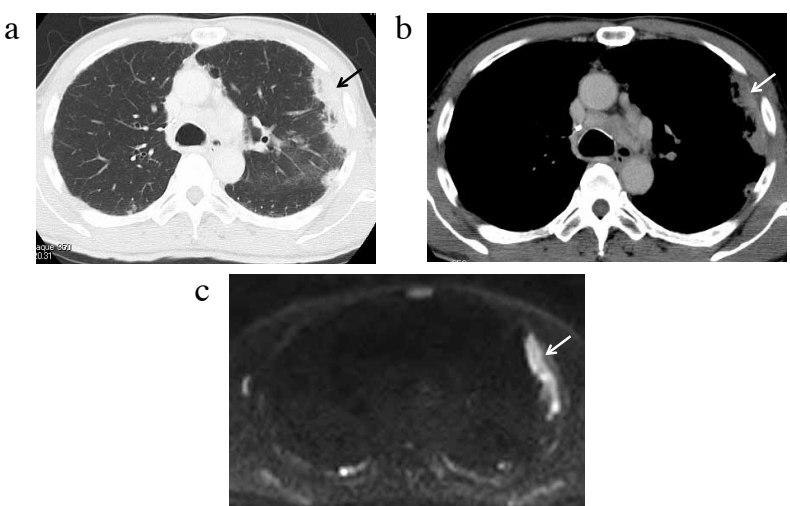

Figure 2. Case 2: A 62 Year-Old Male, Adenocarcinoma, Pulmonary Metastasis in Left Upper Lobe; a: CT (based on lung), b: CT (based on mediastinum), c: DWI, ADC $0.999 \times 10^{-3} \mathrm{~mm}^{2} / \mathrm{sec}$

recognized by decreased diffusion in DWI (Figure 3). Metastatic hilar and mediastinal lymph nodes were clearly represented by decreased diffusion in DWI, and by FDG accumulation in PET-CT (Figure 4). In the CT, it was rather difficult for doctors to recognize the metastatic hilar and mediastinal lymph nodes. Pleural metastasis and nodal involvement were recognized clearly in DWI (Figure 5). Non-malignant organs disappear in DWI. Although it was sometimes difficult to detect liver metastasis by unenhanced CT or PET-CT, it was easy to detect liver metastases by DWI because they were represented by
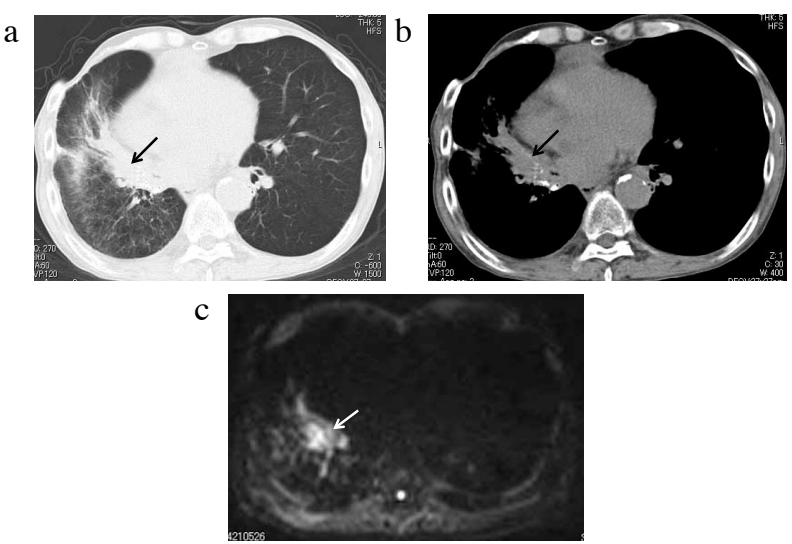

Figure 3. Case 3: A 61 Yaer-Old Male, Squamons Cell Carcinoma, Pulmonary Local Recurrence in Right Middle Lobe; a: CT (based on lung), b: CT (based on mediastinum), c: DWI, ADC $1.164 \times 10^{-3} \mathrm{~mm}^{2} / \mathrm{sec}$
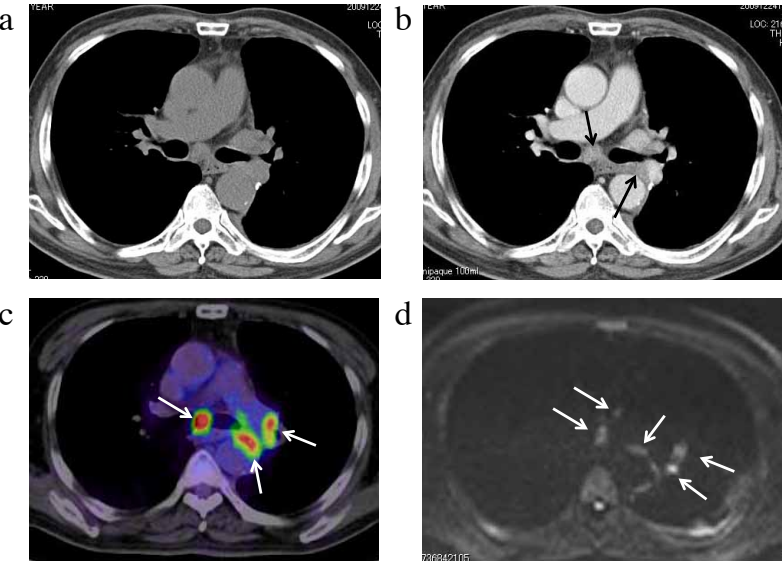

Figure 4. Case 4: A77 Year-Old Male, Adenocarcinoma, Hilar and Mediastinal Lymph Nodes Metastasis; a: CT (based on lung), b: enhanced CT (based on mediastinum), c: PET-CT, SUVmax 10.66, d: DWI, ADC $1.557 \times 10^{-3}$ $\mathrm{mm}^{2} / \mathrm{sec}$
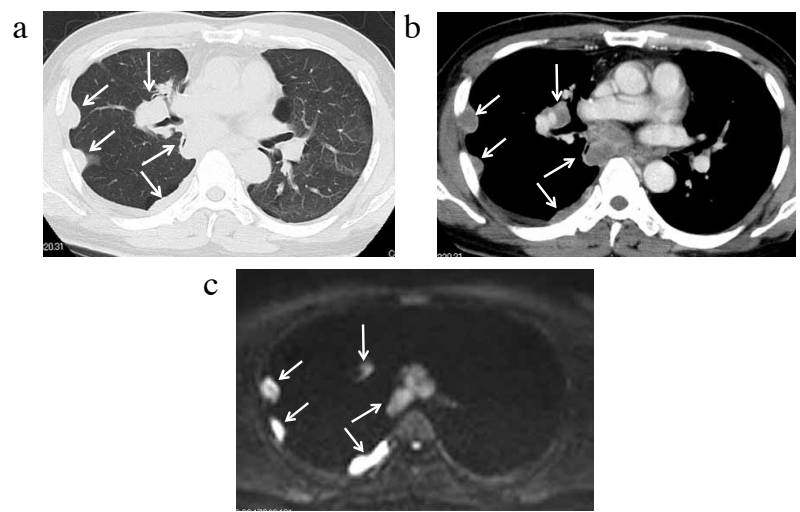

Figure 5. Case 5: 53 Year-Old Male, Pleomorphic Carcinoma, Pleural Metastasis, Hilar and Mediastinal Lymph Nodes Metastasis; a: CT (based on lung), b: enhanced CT (based on mediastinum), c: DWI, ADC $0.973 \times 10^{-3} \mathrm{~mm}^{2} / \mathrm{sec}$

decreased diffusion, different from normal tissue (Figure 6). A female patient with lung cancer with multiple bone metastases and liver metastases is presented in Figure 7. Bone scintigraphy showed multiple bone metastases. 


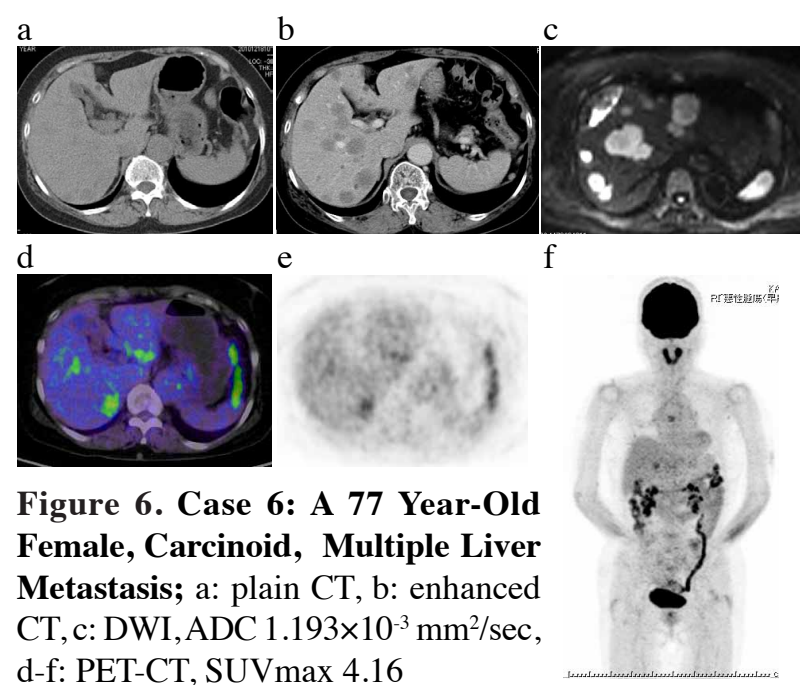

d-f: PET-CT, SUVmax 4.16

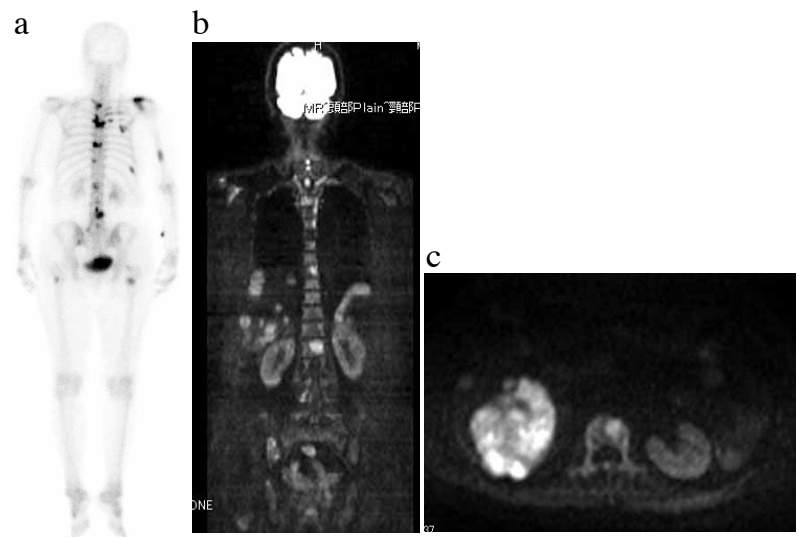

Figure 7. Case 7. A 77 Year-Old Female. Carcinoid, Multiple Bone Metastasis and Multiple Liver Metastasis; a: bone scintigraphy, b, c: DWI, ADC $0.856 \times 10^{-3} \mathrm{~mm}^{2} / \mathrm{sec}$

Table 2. Detection of Recurrence or Metastasis of Lung Cancer

\begin{tabular}{lcccc}
\hline Examinations & \multicolumn{3}{c}{ Detection } & No. of patients \\
& DWI & CT & PET-CT & \\
\hline DWI+CT+PET-CT & + & + & + & 12 \\
$(n=16)$ & + & - & + & 2 \\
& + & + & - & 2 \\
DWI+CT & + & + & & 13 \\
$(n=17)$ & + & - & & 4 \\
DWI+PET-CT & + & & + & 3 \\
$(n=3)$ & & & &
\end{tabular}

+: helpful, -: unhelpful

DWI showed not only multiple bone metastases but also multiple liver metastases.

In the 16 patients who underwent DWI, CT and PET-CT, metastatic lesions were recognized by all these methods in 12 patients, recognized by DWI and PET-CT but not by $\mathrm{CT}$ in 2 patients, and recognized by DWI and CT but not by PET-CT in 2 other patients (Table 2). In the 17 patients who underwent DWI and CT, metastatic lesions were recognized by both methods in 13 patients and recognized by DWI but not by CT in 4 patients. In the 3 patients who underwent DWI and PET-CT, all metastatic lesions were recognized by DWI and PET-CT. On the whole, detection rate of recurrence or metastasis was

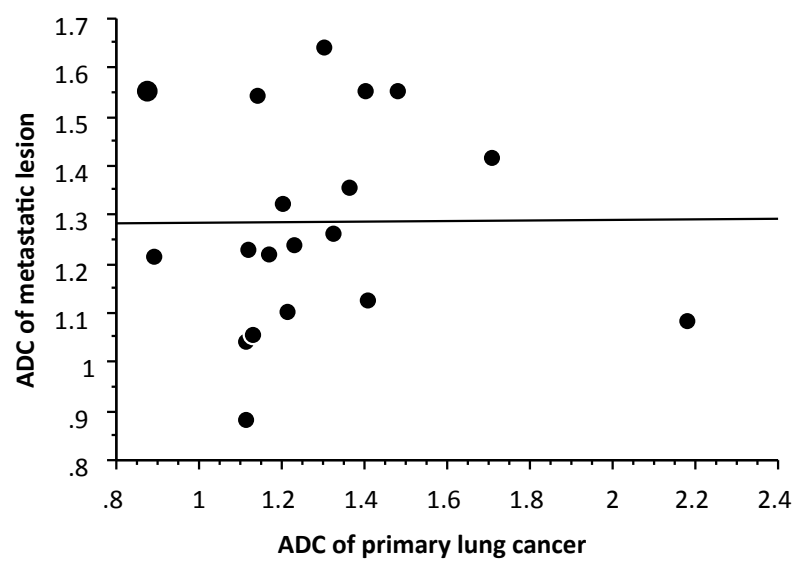

Figure 8. Relationships between ADC of Primary Lung Cancer and ADC of Metastatic Lesion; ADC of metastatic lesion $=1.28+0.005 \times \mathrm{ADC}$ of primary lung cancer. Correlation coefficient: 0.0063

$100 \%(36 / 36)$ in DWI, $89 \%(17 / 19)$ in PET-CT and $82 \%$ $(27 / 33)$ in CT. Detection rate of DWI was significantly higher than that of CT $(\mathrm{p}=0.0244)$ but not significantly higher than that of PET-CT $(p=0.22)$.

ADC values of recurrent or metastatic lesions were $1.31 \pm 0.278$ in lung, $1.36 \pm 0.171$ in lymph nodes, $1.10 \pm 0.149$ in pleura, $1.06 \pm 0.267$ in bone, $1.11 \pm 0.238$ in liver, 0.989 in brain and 1.55 in spleen (Table 1). Increased DWI signals showed every recurrent or metastatic lesion, and could be distinguished from noncancer sites. If the optimal cutoff value (OCV) of DWI for diagnosing malignancy was set to be $1.70 \times 10^{-3} \mathrm{~mm}^{2} / \mathrm{sec}$, the sensitivity of DWI was $95.6 \%$ (43/45). Two metastatic lesions represented higher ADC value over the OCV.

In this series, ADC values of 19 primary lung cancers were obtained from previous MRI records. Comparison between ADC of primary lung cancer and ADC of metastatic lesions are represented in Figure 8. The correlation coefficient of the ADCs was 0.0063, which meant less correlation between ADC of primary lung cancer and ADC of metastatic lesions.

\section{Discussion}

Detection of recurrence or metastasis of lung cancer is an important issue in the follow-up of the cancer treatment. Pulmonary metastases are easy to detect because they are recognized as new lesions. However, they are sometimes rather difficult to distinguish from benign lesions. PET-CT is used in the evaluation of these new lesions. Recently we have reported that DWI is superior to PET in the detection of primary lesions and nodal assessment of non-small cell lung cancers (Usuda et al., 2011). The sensitivity of DWI was significantly higher than that of FDG-PET, and the accuracy of DWI was higher than that of FDG-PET in pulmonary nodules and mass lesions (Usuda et al., 2014). The diagnostic efficacy of DWI is superior to that of PETCT, and DWI can be used in assessment of malignancy in pulmonary nodules and mass lesions instead of PET-CT. Similarly, DWI can be used to distinguish benign from malignant lesions in the lung (Mori et al., 2008; Ohba et al., 2009), in the thorax (Tondo et al., 2011), in the 
prostate (Yamamura et al., 2011), in the breast (Fornasa et al., 2011) and in the liver (Koike et al., 2009). DWI was reported to be an effective alternative in future diagnosis and follow-up of bladder tumors because of its higher sensitivity, specificity and accuracy (Ceylan K et al., 2010). Preoperative prediction of neurovascular bundle involvement of localized prostate cancer was reported to be possible by combined T2 and DWI, number of positive biopsy cores and Gleason score (Naiki et al., 2011).

Metastases of hilar and mediastinal lymph nodes are detected by CT or FDG-PET, but tiny metastatic lymph nodes are difficult to be detected by CT or FDG-PET, and it is sometimes difficult for CT or FDG-PET to distinguish malignant lesions from benign lesions. DWI was reported to be able to detect more tiny metastatic lymph nodes than that detected by FDG-PET (Usuda et al., 2013). In the paper the maximum diameter of metastatic lymph nodes were $7.2 \pm 4.1 \mathrm{~mm}$ in 39 detected by DWI and $11.9 \pm 4.1$ $\mathrm{mm}$ in 24 detected by FDG-PET.

Metastasis of the liver is sometimes difficult to detect by unenhanced CT or FDG-PET. However, DWI was very useful in the detection of liver metastasis, which had distinguishable decreased diffusion different from benign lesions. It has been reported that MRI has an extremely high degree of sensitivity in the detection of colorectal liver metastasis (Blyth et al., 2008).

Whole-body MRI with DWI can be used for bone metastasis assessment of non-small cell lung cancer patients; which is as accurate as bone scintinography and/or PET-CT (Takenaka et al., 2009). PET-CT appears to be slightly less sensitive than whole body MRI in the detection of osteal metastases (Ghanem et al., 2005). Already a trial of whole-body simultaneous hybrid PET/MRI was performed for neoplastic lesions, and simultaneous PET/MRI is a promising technology for the detection of neoplastic disease (Rakheja et al., 2013).

In the comparison between ADC of primary lung cancer and ADC of metastatic lesions, it was surprised for us that the correlation coefficient was rather low, which meant that cellular density of cancer cells would change a lot from that of the primary lung cancer because DWI represent the number and distribution of cancer cells (Usuda et al. 2013). It may be possible to assess the pathological characteristics of metastatic lesions of primary lung cancers using DWI.

DWI was effective in detecting recurrence or metastasis of lung cancers, especially of the metastasis of hilar or mediastinal lymph nodes, liver, or bone which was rather difficult to distinguish from benign lesions by CT. DWI is useful for the detection of recurrence and metastasis of lung cancer.

\section{Acknowledgements}

This study was supported by a Grant-in-Aid for Scientific Research from the Ministry of Education, Culture, Sports, Science and Technology, Japan (25462186). We are grateful to Mr. Masaru Takahashi and Mr. Keiya Hirata of the MRI Center, The Medical University, for technical assistance.

\section{References}

Blyth S, Blakeborough A, Peterson M, Cameron IC, Majeed AW (2008). Sensitivity of magnetic resonance imaging in the detection of colorectal liver metastases. Ann R Coll Surg Engl, 90, 25-8.

Ceylan K, Taken K, Gecit I, et al (2010). Comparison of cystoscopy with diffusion-weighted magnetic resonance images used in the diagnosis and follow-up of patients with bladder tumors. Asian Pac J Cancer Prev, 11, 1001-4.

Fornasa F, Pinali L, Gasparini A, Toniolli E, Montemezzi S (2011). Diffusion-weighted magnetic resonance imaging in focal breast lesions. Analysis of 78 cases with pathological correlation. Radiol med, 116, 264-75.

Ghanem N, Uhl M, Brink I, et al (2005). Diagnostic value of MRI in comparison to scintigraphy, PET, MS-CT and PET/CT for the detection of metastases of bone. Eur J Radiol, 55, 41-55.

Koike N, Cho A, Nasu K, et al (2009). Role of diffusion-weighted magnetic resonance imaging in the differential diagnosis of focal hepatic lesions. World J gastroenterol, 15, 5805-12.

Le Bihan D, Breton E, Lallemand D, et al (1988). Separation of diffusion and perfusion in intravoxel incoherent motion MR imaging. Radiology, 168, 497-505.

Mori T, Nomori H, Ikeda K, et al (2008). Diffusion-weighted magnetic resonance imaging for diagnosing malignant pulmonary nodules/masses. Comparison with positron emission tomography. J Thoracic Oncol, 3, 358-64.

Naiki T, Okamura T, Nagata D, et al (2011). Preoperative prediction of neurovascular bundle involvement of localized prostate cancer by combined $\mathrm{T} 2$ and diffusion-weighted imaging of magnetic resonance imaging, number of positive biopsy cores and Gleason score. Asian Pac J Cancer Prev, 12, 909-13.

Ohba Y, Nomori H, Mori T, et al (2009). Is diffusion-weighted magnetic resonance imaging superior to positron emission tomography with fludeoxyglucose $\mathrm{F} 18$ in imaging non-small cell lung cancer? J Thorac Cardiovasc Surg, 138, 439-45.

Rakheja R, Chandarana H, DeMello L, et al (2013). Correlation between standardized uptake value and apparent diffusion coefficient of neoplastic lesions evaluated with whole-body simultaneous hybrid PET/MRI. Am J Roentgenol, 201, 1115-9.

Sorensen AG, Buonanno FS, Gonzalez RG, et al (1996). Hyperacute stroke. Evaluation with combined multisection diffusion-weighted and hemodynamically weighted echoplanar MR imaging. Radiology, 199, 391-401.

Szafer A, Zhong J, Gore JC (1995). Theoretical model for water diffusion in tissues. Magn Reson Med, 33, 697-712.

Takahara T, Imai Y, Yamashita T, (2004). Diffusion weighted whole body imaging with background body signal suppression (DWIBS). Technical improvement using free breathing, STIR and high resolution 3D display. Radiat Med, 22, 275-82.

Takenaka D, Ohno Y, Matsumoto K, et al (2009). Detection of bone metastases in non-small cell lung cancer patients: comparison of whole-body diffusion-weighted imaging (DWI), whole-body MR imaging without and with DWI, whole-body FDG-PET/CT, and bone scintigraphy. J Magn Reson Imaging, 30, 298-308.

Tien RD, Felsberg GJ, Friedman H, Brown M, MacFall J (1994). MR imaging of high-grade cerebral gliomas. Value of diffusion-weighted echoplanar pluse sequences. AJR. 162, 671-7.

Tondo F, Saponaro A, Stecco A, et al (2011). Role of diffusionweighted imaging in the differential diagnosis of benign and malignant lesions of the chest-mediastinum. Radiol Med, 116, 720-33. 
Katsuo Usuda et al

Usuda K, Zhao XT, Sagawa M, et al (2011). Diffusion-weighted imaging is superior to PET in the detection and nodal assessment of lung cancers. Ann Thorac Surg, 91, 1689-95.

Usuda K, Sagawa M, Motono N, et al (2013). Advantages of diffusion-weighted imaging over positron emission tomography-computed tomography in assessment of hilar and mediastinal lymph node in lung cancer. Ann Surg Oncol, 20, 1676-83.

Usuda K, Zhao XT, Sagawa M, et al (2013). Diffusion-weighted imaging (DWI) signal intensity and distribution represent the amount of cancer cells and their distribution in primary lung cancer. Clinical Imaging, 37, 265-72.

Usuda K, Sagawa M, Motomo N, et al (2014). Diagnostic performance of diffusion weighted imaging of malignant and benign pulmonary nodules and masses. Comparison with positron emission tomography. Asian Pac J Cancer Prev, 15, 4629-35.

Yamamura J, Salomon G, Buchert R, et al (2011). Magnetic resonance imaging of prostate cancer. Diffusion-weighted imaging in comparison with sextant biopsy. J Comput Assist Tomog, 35, 223-8. 